\title{
Rheological Behavior of Concentrated Hyperbranched Polymer/Silica Nanocomposite Suspensions
}

\author{
Valérie Geiser, Yves Leterrier,* and Jan-Anders E. Månson \\ Laboratoire de Technologie des Composites et Polymères (LTC), Ecole Polytechnique Fédérale de Lausanne \\ (EPFL), CH-1015 Lausanne, Switzerland
}

Received March 15, 2010; Revised Manuscript Received July 8, 2010

\begin{abstract}
The rheological behavior of two hyperbranched polymer/silica suspensions with different dispersion states, surface chemistries, and concentrations of the silica nanoparticles was investigated in terms of viscoelastic properties, activation energy for viscous flow, and yield stress. The viscoelastic properties of both types of suspensions were reduced to a master curve that was a function of the limiting viscosity and shear modulus. A liquid-to-solid transition and correlated activation energy change were found to occur for particle volume fraction in the range of $5-10 \%$ for well-dispersed systems and $20-25 \%$ for systems where silylated particles were agglomerated. The viscosity of the suspensions was found to be considerably higher than that predicted by the classical percolation model for concentrated particle suspensions; this was argued to result from an immobilized layer of polymer on the surface of the silica particles. The percolation model was therefore modified to include such confined layer in order to predict the viscosity as a function of filler fraction. In the case of silylated particles with weak interactions with the polymer, the model based on an immobilized layer of thickness in the range of $2-5 \mathrm{~nm}$ reproduced the data. In the case of well-dispersed particles with strong interfacial interactions, the immobilized layer was correlated to the average distance between adjacent particles. In this case the model predicted an exponential increase of the viscosity with particle fraction and that the whole matrix gelled at particle concentrations larger than 5 vol \%, corresponding to a $7.5 \mathrm{~nm}$ thick immobilized layer.
\end{abstract}

\section{Introduction}

Polymer nanocomposites with inorganic nanoparticles ${ }^{1-4}$ or carbon nanotubes ${ }^{5,6}$ have motivated numerous studies owing to their outstanding thermomechanical properties. It was shown that, for the same filler loading, nanosized fillers have a much higher impact on reinforcement than microsized fillers ${ }^{7-11}$ as a consequence of the extremely large specific interfacial area and very short distance between reinforcing particles. However, the claimed benefits of nanocomposites rely on a good dispersion of the particles, usually associated with processing problems. In fact, small amounts of nanoparticles drastically alter the viscoelastic properties of the material, transforming the liquidlike polymer into a solidlike composite paste. ${ }^{12-15}$ The liquid-to-solid transition is a major challenge for nanocomposite processing and is often overcome with the use of solvents. The critical volume fraction, beyond which the viscosity diverges toward infinity, is $\phi^{*} \sim 0.64$ for random close-packed spherical particles at low shear rate ${ }^{16}$ and $\sim 0.7$ at high shear rate. However, the liquid-tosolid transition for nanoparticle suspensions was found to occur at a particle fraction as low as $10 \% .^{17,18}$ Several studies ${ }^{13,19,20}$ concluded that this low $\phi^{*}$ was observed for sufficiently strong interfacial interactions, resulting in an immobilized layer adsorbed on the surface of the particles and in confinement effects between particles. The degree of immobilization as well as the thickness of the confined layer was correlated to the affinity between matrix and particles. Because of the high specific surface area of nanoparticles, the fraction of confined matrix can be quite large; hence the strong increase in viscosity. As an example, a 200 -fold increase in viscosity was found when $3.4 \mathrm{wt} \%$ fumed

*Corresponding author. E-mail: yves.leterrier@epfl.ch. silica was added to a cyanate ester. ${ }^{21}$ Similar findings were reported for entangled ${ }^{22}$ and nonentangled ${ }^{23}$ polymer melts.

The present work is devoted to the case of hyperbranched polymers $^{24}$ (HBP), which would a priori pertain to the nonentangled case, the difference being essentially the much higher molecular weight and size of HBP compared to nonentangled oligomers. HBP are macromolecules characterized by a highly branched structure and multiplicity of reactive chain ends. They belong to the family of dendritic polymers ${ }^{25}$ but are less perfectly branched than the monodisperse dendrimers. HBP are synthesized in a rapid one-step process that makes them an attractive low-cost alternative to dendrimers produced in a multistep process. The chemical, physical, and rheological properties of HBP and dendrimers have been investigated in several studies. ${ }^{24,26-28}$ This class of macromolecules have very distinct rheological properties; i.e., they show low Newtonian viscosity even at high molecular weight, owing to their globular structure and absence of entanglements. This behavior was exploited through the use of HBP as lowviscosity toughening additives to thermosets ${ }^{29,30}$ and should also be useful in postponing the liquid-to-solid transition to higher particle loadings for HBP-based nanocomposites. Another interesting feature of HBP is their low shrinkage ${ }^{31}$ upon cross-linking and related low internal stress in the solid state compared to standard resins. ${ }^{32,33}$ Moreover, the addition of solid particles has been shown to further reduce polymerization shrinkage. ${ }^{34}$

The aim of the present work was to determine the influence of spherical nanoparticles on the rheology of HBP with particle loadings up to the concentrated regime. Attention was paid to the occurrence of a liquid-to-solid transition and related polymer immobilization effects on the surface of the particles. An acrylated HBP and two different nanosized silica particles, leading to different dispersion morphologies, were selected. 


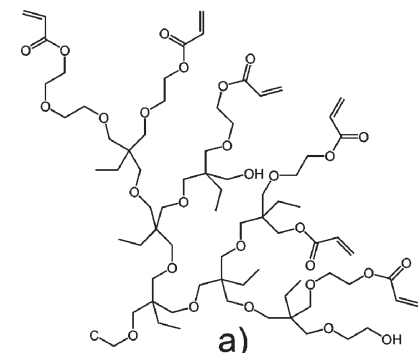

a)

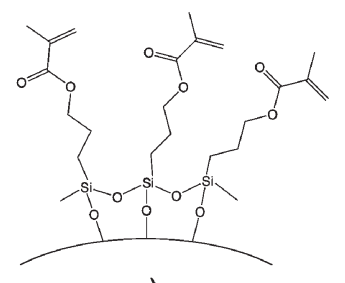

c)
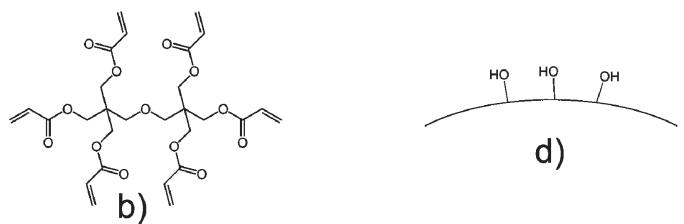

d)

Figure 1. Molecular structures of (a) acrylated hyperbranched polymer (C denotes the core of the molecule and only one of the four branches is shown), (b) dipentaerythritol hexaacrylate, (c) silylated Aerosil surface (three methacrylsilane molecules are shown), and (d) silanol Highlink surface.

\section{Materials and Methods}

Materials. The monomer was based on a third-generation hyperbranched polyether polyol, giving a 29-functional hyperbranched polyether acrylate depicted in Figure 1 (Perstorp AB, Sweden). ${ }^{34}$ Several tests were also performed with another acrylated monomer (dipentaerythritol hexaacrylate, DPHA, Sigma-Aldrich) also shown in Figure 1. Two amorphous silica nanoparticles were used, and their surface chemistry is shown in Figure 1. Highlink NanO G502 (Clariant) is a suspension of $30 \mathrm{wt} \%$ monodispersed $\mathrm{SiO}_{2}$ in isopropanol. These organosols were found to be slightly more hydrophobic than aqueous silica sols. ${ }^{35}$ The average particle size according to the supplier was $13 \mathrm{~nm}$ (BET measurements gave a size of $12 \mathrm{~nm}^{35}$ ), which corresponds to a specific surface area of about $220 \mathrm{~m}^{2} / \mathrm{g}$. Aerosil $\mathrm{R} 7200$ (Degussa) is a $\mathrm{SiO}_{2}$ powder of aggregated particles with a specific surface area of about $150 \mathrm{~m}^{2} / \mathrm{g}$ and a primary particle size of $12 \mathrm{~nm}$. Aerosil particles were subjected to a surface treatment with methacrylsilane.

Highlink and Aerosil suspensions containing up to $25 \mathrm{vol} \%$ (38 wt \%) $\mathrm{SiO}_{2}$ in the acrylated HBP were prepared by ultrasonic mixing of the silica in isopropanol, then mixing of the suspension with the HBP, and subsequent solvent removal. Details of sample preparation are given elsewhere. ${ }^{34}$ As shown in transmission electron micrographs (TEM, Philips/FEI, $\mathrm{CM} 12,120 \mathrm{kV}$ ) of cured materials in Figure 2, the composites containing Highlink particles were true nanocomposites, where the inorganic phase was monodispersed in the polymer matrix. In contrast, the Aerosil powder could not be completely disagglomerated during the ultrasound treatment. Image analysis revealed that the average agglomerate size for the Aerosil suspensions was $120 \mathrm{~nm}$ with a very large size distribution. The volume fraction of $\mathrm{SiO}_{2}$ in the agglomerates $\left(\phi_{\mathrm{agg}}\right)$ was found to be equal to $55 \%$ at $5 \mathrm{vol} \% \mathrm{SiO}_{2}$. For higher volume fractions image analysis was difficult due to overlapping agglomerates, but $\phi_{\text {agg }}$ did not seem to change significantly.

Rheology. Viscosity and shear modulus measurements were carried out on a strain-controlled rotational rheometer (ARES, Rheometric Scientific, 2kFRT transducer) equipped with a temperature-controlled oven, using a cone-plate geometry with a diameter of $25 \mathrm{~mm}$, a cone angle of $0.1 \mathrm{rad}$, and a gap of 0.051 $\mathrm{mm}$ in dynamic mode. Strain was ensured to be in the linear viscoelastic range at any frequency and temperature, i.e., between 0.1 and $30 \%$ depending on the composition.
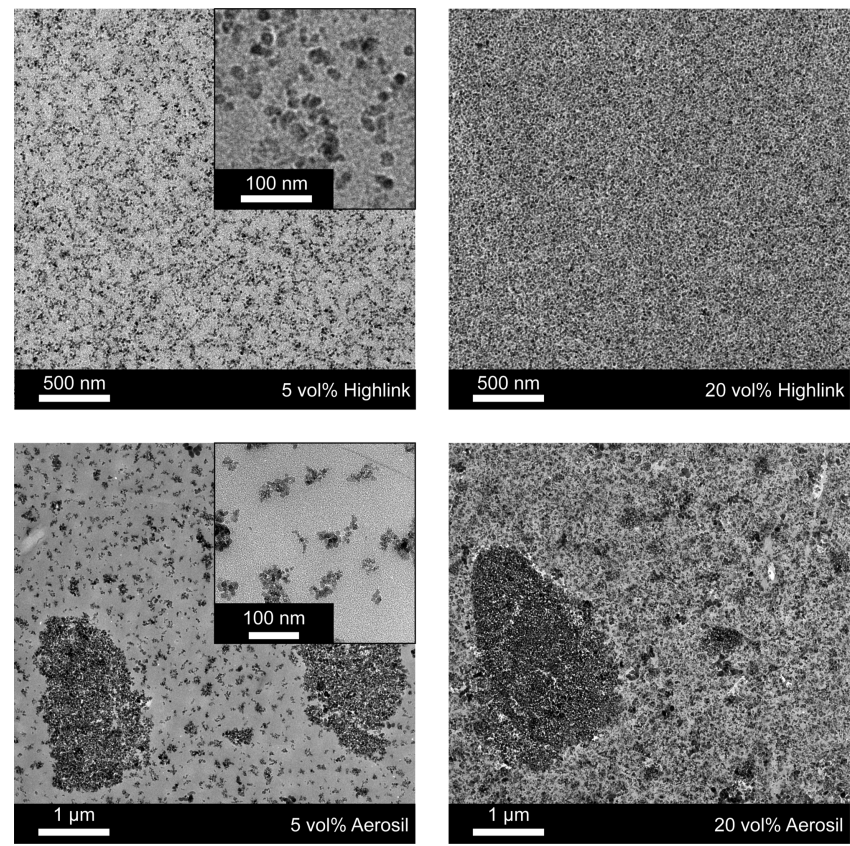

Figure 2. Transmission electron micrographs of Highlink and Aerosil composites at 5 and $20 \mathrm{vol} \%$ silica particle fraction.

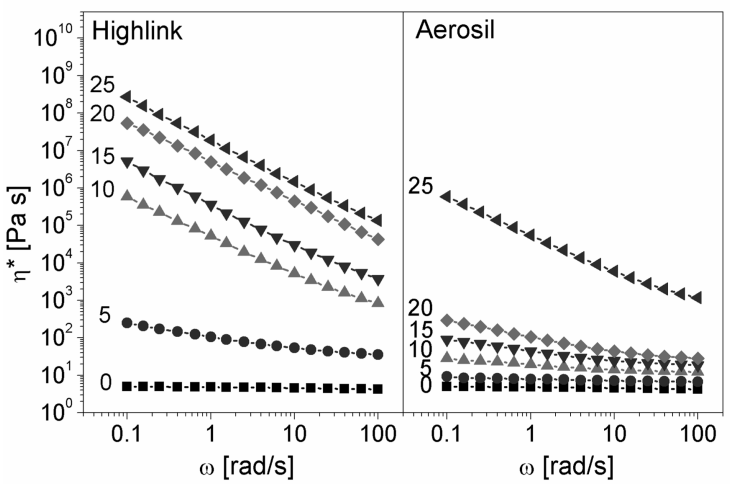

Figure 3. Viscosity of $\mathrm{HBP} /$ silica suspensions as a function of frequency and particle volume fraction from 0 to $25 \%$ (as indicated).

\section{Results}

Figure 3 depicts the viscosity as a function of frequency $\omega$ and $\mathrm{SiO}_{2}$ volume fraction $\phi$ for the Highlink and Aerosil suspensions in the acrylated HBP. The pure HBP showed Newtonian behavior with viscosity independent of frequency in the investigated range. The viscosity increased with increasing filler fraction with shear thinning becoming apparent at $\phi=5 \%$ for Highlink and at $\phi=10 \%$ for Aerosil. Shear thinning was the result of an increase in relaxation time with respect to the pure polymer. The increase in viscosity as well as the shear thinning behavior with increasing filler fraction was much more pronounced for Highlink than for Aerosil. At a volume fraction of $10 \%$, well-dispersed Highlink increased the HBP viscosity at $0.1 \mathrm{rad} / \mathrm{s}$ by more than 5 orders of magnitude. At $25 \mathrm{vol} \%$ the increase reached 8 orders of magnitude, whereas it was 1000 times less for agglomerated Aerosil. Such huge increases in viscosity far exceeded previously reported data for concentrated suspensions of hard spheres in Newtonian fluids, ${ }^{23,36,37}$ which implies that the present acrylated HBP presented a very strong affinity with the silica sol (Highlink) and to a lesser extent with silylated particles (Aerosil). These results moreover demonstrate the considerable influence of the 


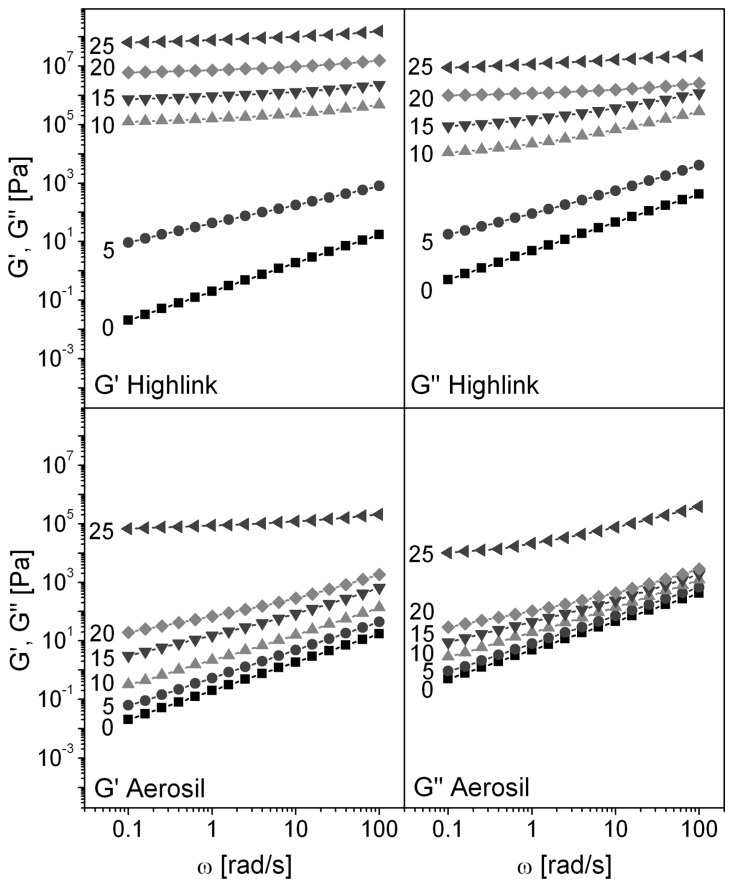

Figure 4. Shear moduli of $\mathrm{HBP} /$ silica suspensions as a function of frequency and particle volume fraction from 0 to $25 \%$ (as indicated).

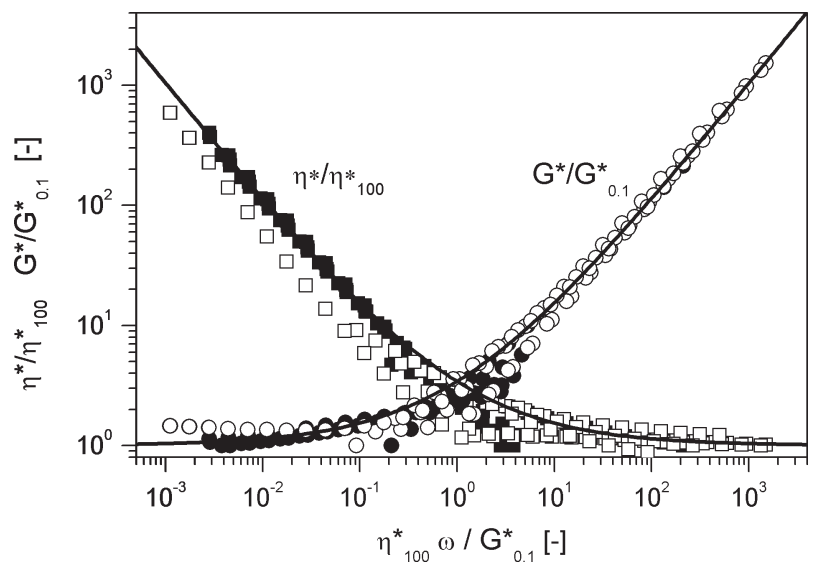

Figure 5. Superposition of reduced rheological data vs reduced frequency. Open symbols: Aerosil; closed symbols: Highlink. The solid lines represent eq 1 with $\alpha=0.47$ and $\kappa=1.09$.

interfacial interactions and dispersion state of nanoparticles on the viscosity of concentrated HBP suspensions.

Shear thickening was not detected in the investigated frequency domain. This phenomenon results from a temporary hydrodynamic clustering of compact groups of particles formed as shear forces drive them into contact. ${ }^{38}$ It was reported to occur above a critical shear rate, usually in the range from 1 to $10 \mathrm{~s}^{-1}$, where short-range lubrication forces dominate the observed viscosity increase (e.g., ref 39). The critical shear rate $\dot{\gamma}_{\text {crit }}$ corresponds to a critical Péclet number, $P e_{\text {crit }}=6 \pi \eta \dot{\gamma}_{\text {crit }} r^{3} / k_{\mathrm{B}} T$ ( $\eta$ is the viscosity of the continuous phase, $r$ the particle radius, $k_{\mathrm{B}}$ the Boltzmann constant, and $T$ the absolute temperature) larger than several $10 .^{40} \mathrm{~A}$ minimum strain amplitude is also required for suspensions to shear thicken. ${ }^{41}$ Shear thickening of Aerosil suspensions in polypropylene glycol was indeed found to occur at dynamic shear rates $\gamma_{0} \omega$ (where $\gamma_{0}$ is the strain amplitude and $\omega$ is the frequency) above $10 \mathrm{~s}^{-1}$. ${ }^{2}$ The investigated suspensions were tested at shear rates below $3 \mathrm{~s}^{-1}$, i.e., below the critical conditions $\left(P e_{\text {crit }}\right.$ was smaller than 0.15 in the case of Highlink and smaller

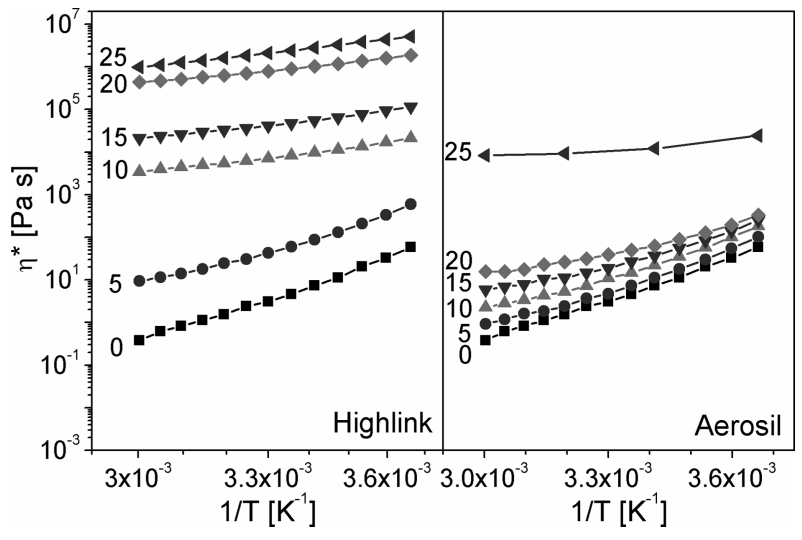

Figure 6. Viscosity of $\mathrm{HBP} / \mathrm{silica}$ suspensions at $\omega=6.28 \mathrm{rad} / \mathrm{s}$ as a function of inverse temperature and particle volume fraction from 0 to $25 \%$ (as indicated).

than 21 in the case of Aerosil aggregates, which again is below the critical condition, $P e>100$, even at the highest investigated shear rate of $3 \mathrm{~s}^{-1}$ ).

Figure 4 shows the complex shear moduli $G^{\prime}$ and $G^{\prime \prime}$ as a function of frequency and $\mathrm{SiO}_{2}$ fraction for the two types of suspensions. For the pure HBP, $G^{\prime}$ and $G^{\prime \prime}$ were both approximately proportional to $\omega$, indicating that the polymer was not exactly a Newtonian fluid for which $G^{\prime}=0$ and $G^{\prime \prime} \sim \omega$. In fact, since $G^{*}=\eta^{*} \omega=\left(G^{\prime 2}+G^{\prime \prime 2}\right)^{1 / 2}$ was dominated by $G^{\prime \prime}$, the HBP could be considered as a viscoelastic liquid with Newtonian-like behavior. At increasing $\mathrm{SiO}_{2}$ fraction the scaling progressively evolved toward that for an elastic solid.

Assuming that the low-strain rheological response was dominated by a single relaxation time $\eta_{\infty}^{*} / G_{0}^{*}$, where $G_{0}^{*}(\phi)$ is the limiting modulus as $\omega \rightarrow 0$ and $\eta_{\infty}^{*}(\phi)$ is the limiting viscosity as $\omega \rightarrow \infty$, the dimensionless quantities $G^{*} / G_{0}^{*}$ and $\eta^{*} / \eta_{\infty}^{*}$ were described by a single master curve when plotted against the dimensionless frequency $\omega \eta_{\infty}^{*} / G_{0}^{*}$ :

$$
\begin{aligned}
& \frac{G^{*}}{G_{0}^{*}} \approx 1+\left(\frac{\eta_{\infty}^{*} \omega}{G_{0}^{*}}\right)+\kappa\left(\frac{\eta_{\infty}^{*} \omega}{G_{0}^{*}}\right)^{\alpha} ; \\
& \frac{\eta^{*}}{\eta_{\infty}^{*}} \approx 1+\left(\frac{\eta_{\infty}^{*} \omega}{G_{0}^{*}}\right)^{-1}+\frac{1}{\kappa}\left(\frac{\eta_{\infty}^{*} \omega}{G_{0}^{*}}\right)^{-\alpha}
\end{aligned}
$$

where $\kappa$ and $\alpha$ are parameters. This scaling was used by Rodlert et al. ${ }^{14}$ based on Lin's ${ }^{43}$ model for steady state shear thinning particle suspensions, to which belong the investigated nanocomposites. Since no plateau values for $G_{0}^{*}(\phi)$ and $\eta_{\infty}^{*}(\phi)$ could be observed for the shear thinning mixtures in the investigated frequency range, the corresponding values at $\omega=0.1 \mathrm{rad} / \mathrm{s}$ and $\omega=100 \mathrm{rad} / \mathrm{s}$, respectively, were chosen, and the limiting data were called $G_{0.1}^{*}$ and $\eta_{100}^{*}$. The reduced data are shown in Figure 5 together with fitted eq 1 using $\kappa=1.09$ and $\alpha=0.47$. These values are very close to the values that Rodlert found for hyperbranched polymer/clay nanocomposites. Interestingly, eq 1 accounts well for both suspension families, and it is therefore inferred that the rheological data of any suspension may be expressed entirely in terms of the parameters $G_{0.1}^{*}$ and $\eta_{100}^{*}$.

Figure 6 shows the influence of temperature $T$ on the viscosity of $\mathrm{HBP}$ and $\mathrm{HBP} /$ particle suspensions in so-called Arrhenius coordinates. As temperature increased, the viscosity of the suspensions was reduced due to the temperature dependence of the HBP viscosity. For both types of particles this trend became less pronounced with increasing fraction, indicating that the suspensions became independent of the matrix viscosity and that 


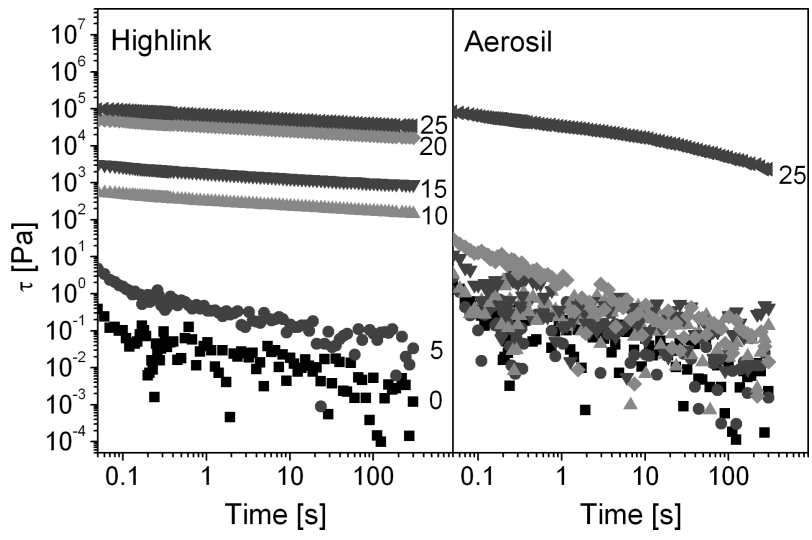

Figure 7. Relaxation of the stress $\tau$ under $1 \%$ shear deformation of $\mathrm{HBP} /$ silica suspensions as a function of particle volume fraction from 0 to $25 \%$ (as indicated).

a temperature-independent rigid network had formed. ${ }^{14}$ As was already noticed in Figure 4, the Highlink suspensions became considerably stiffer than the Aerosil suspensions with increasing particle fraction.

A further insight into the viscoelastic properties of the HBP/ silica suspensions was obtained from stress relaxation experiments. Figure 7 shows the stress resulting from the application of a $1 \%$ shear deformation. The residual stress corresponds to the yield stress of the material, ${ }^{44}$ i.e., the minimum stress necessary to deform a yield stress fluid. The scatter at low filler fraction was due to the sensitivity limit of the rheometer. The stress and relaxation time both increased with increasing filler fraction, which was consistent with the emergence of shear thinning shown in Figure 3. A power-law relaxation behavior was moreover evident, especially for Highlink suspensions with an exponent equal to -0.13 for all concentrations beyond $10 \mathrm{vol} \%$. The present evidence for self-similar dynamics implies that the concentrated $\mathrm{HBP} /$ silica suspensions behave as a network-type fluid similar to elastomers ${ }^{45}$ and entangled ring-type polymers. ${ }^{46}$

The above results are synthesized in the composite Figure 8, which represents different facets of the liquid-to-solid transition of the HBP/silica suspensions. Figure 8a shows the tangent of the phase angle $\tan (\delta)=G^{\prime \prime} / G^{\prime}$, obtained from Figure 4, which is a measure for the elasticity of the fluid. $\operatorname{Tan}(\delta)=1$ was used to define the transition from a viscoelastic liquid to a viscoelastic solid and is comparable to physical gelation, where the particles show strong enough interactions with each other to form a 3-dimensional network. For the Highlink suspensions the liquid-to-solid transition occurred at $5 \%<\phi<10 \%$, after which the dependence of $\tan (\delta)$ on the solid phase fraction was weak. The solid nanoparticles formed a network, which conferred to the material a rather elastic behavior. The decrease in $\tan (\delta)$ for the Aerosil suspensions was more gradual, and the liquid-to-solid transition occurred only at $\phi>20 \%$. The same critical volume fractions also appear when looking at the activation energy $E_{\mathrm{A}}$ for viscous flow reproduced in Figure $8 \mathrm{~b}$. These values were calculated from a linear fit of $\ln \left(\eta^{*}\right)$ vs $1 / T$ shown in Figure 6 , where an Arrhenius behavior was evident. For the pure HBP, $E_{\mathrm{A}}$ was equal to $61 \mathrm{~kJ} \mathrm{~mol}^{-1}$. For Highlink suspensions the activation energy dropped at $5 \%<\phi<10 \%$ and then became almost independent of $\phi$. Hence, at $\phi>5 \%$ the viscosity of the composite became independent of the matrix viscosity and therefore independent of the temperature. For Aerosil suspensions the activation energy decreased gradually with the particle fraction and reached the value of the Highlink plateau at $\phi=25 \%$. A similar decrease in activation energy with filler fraction was found by Rodlert et al. ${ }^{47} \mathrm{~A}$ further indication of the liquid-to-solid transition was derived from the stress data (Figure 7), shown in

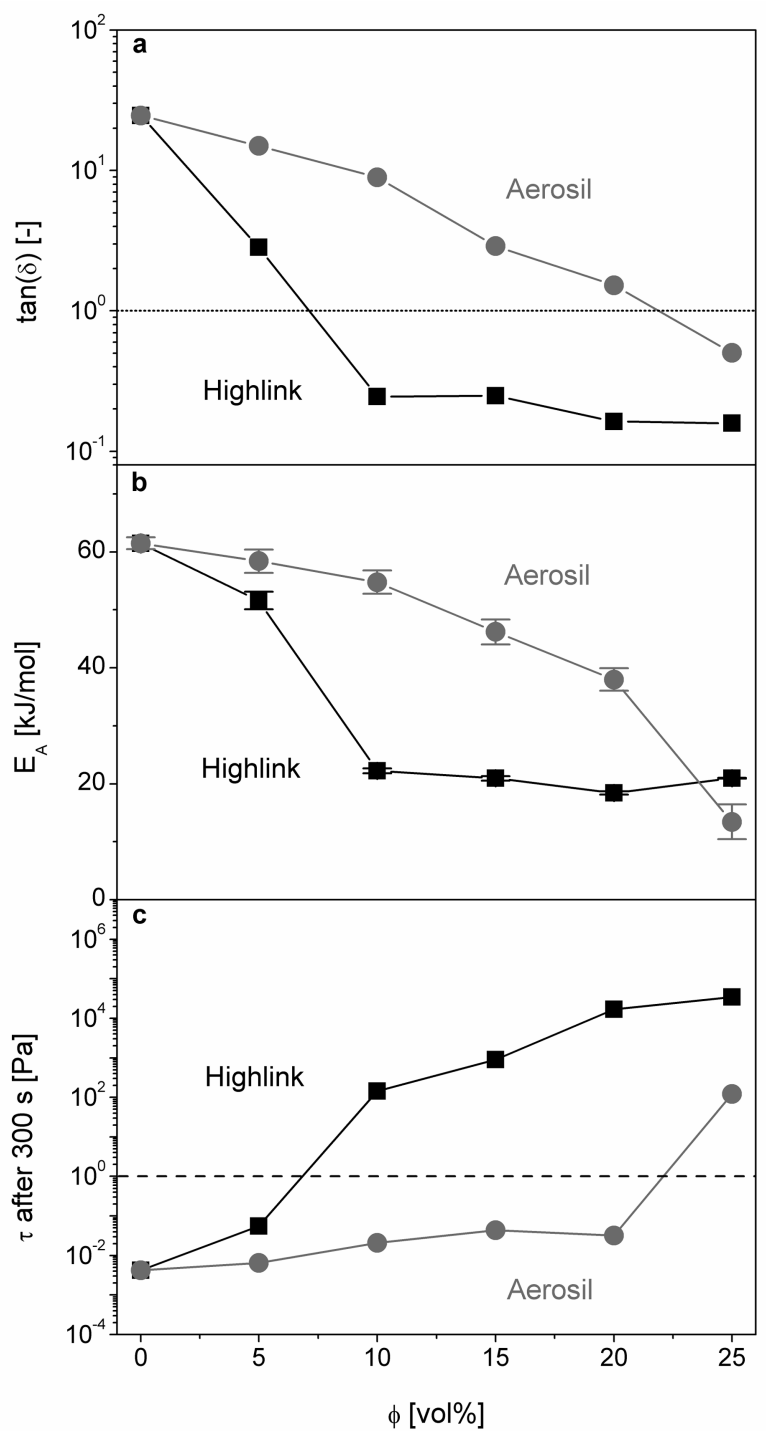

Figure 8. (a) Tangent of the phase angle of HBP/silica suspensions vs particle volume fraction at $\omega=6.28 \mathrm{rad} / \mathrm{s}$ (the dotted line represents the liquid-to-solid transition), (b) activation energy for viscous flow, and (c) stress after $300 \mathrm{~s}$ of relaxation (the dashed line separates the yield-stress fluids from the non-yield-stress fluids).

Figure $8 \mathrm{c}$ after a relaxation time of $300 \mathrm{~s}$. According to Jing et al., ${ }^{48}$ a liquid is considered to be a yield-stress fluid if the residual stress is higher than $1 \mathrm{~Pa}$. For the Highlink suspensions this was the case for $\phi>5 \%$, whereas Aerosil suspensions reached the yield limit only at $\phi>20 \%$.

\section{Discussion}

The huge increase in viscosity of the present HBP suspensions (Figure 3), especially for well-dispersed silica sols, and related early gelation was somehow surprising, as the low viscosity, nonentangled HBP was expected to maintain also a low suspension viscosity. The viscosity of particle suspensions has been analyzed and modeled since early studies made by Einstein, ${ }^{49}$ as summarized in the review of Bicerano et al. ${ }^{50}$ Three different concentration regimes are usually identified for particle suspensions. In the dilute regime with noninteracting particles the viscosity of the suspension is often described in terms of virial expansions (i.e., power series) of the particle volume fraction $\phi$, becoming proportional to $\phi$ in the limit $\phi \rightarrow 0 .{ }^{49}$ Classical models for dilute suspensions were established for particles of sizes larger than several micrometers. ${ }^{51-53}$ These models often underestimate 


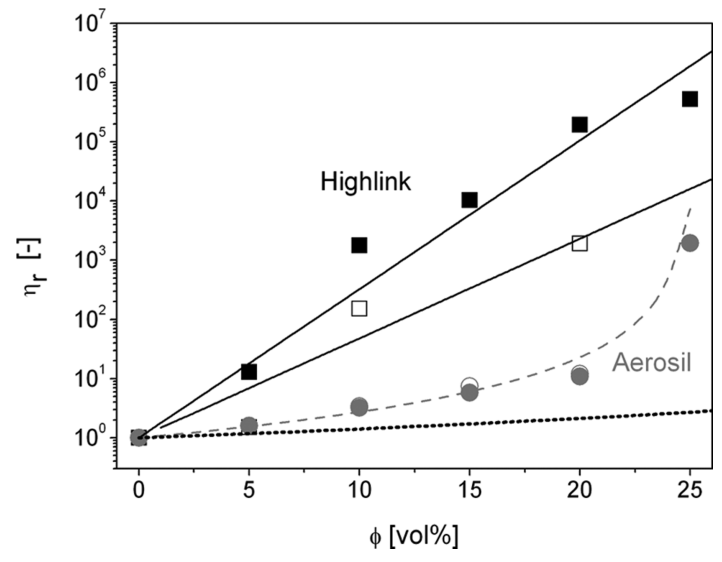

Figure 9. Reduced viscosity of $\mathrm{HBP} /$ silica suspensions (closed symbols) and DPHA/silica suspensions (open symbols) at $\omega=6.28 \mathrm{rad} / \mathrm{s}$ as a function of particle volume fraction. The dotted line represents eq 2 with $K=1, \lambda=2$, and $\phi^{*}=0.64$, the dashed line represents eq 5 with $a=$ $3.3 \mathrm{~nm}, r=9.1 \mathrm{~nm}$, and $\phi^{*}=0.64$, and the solid lines represent eq 8 with $A=29$ for the HBP/Highlink suspensions and $A=19$ for the DPHA/ Highlink suspensions.

the viscosity of nanocomposites, including the present materials by several orders of magnitude, because they do not take into account the very large specific surface area of the particles and possible polymer-particle interactions. The semidilute regime is defined to include the broad range of volume fractions between which the interparticle interactions first become appreciable and at which they begin to predominate. The "crossover volume fraction" defines the transition between the dilute and the semidilute regimes and occurs at $\phi=1-2 \%{ }^{50}$ for spherical particles. The model of "sticky spheres" short-range attractive interactions between particles in the semidilute regime. A suspension is defined as concentrated for volume fractions greater than the geometrical percolation threshold $\phi_{\mathrm{c}}{ }^{50}$ Motions in dispersions with $\phi>\phi_{\mathrm{c}}$ are necessarily collective, but the suspension continues to be fluidlike until interparticle interactions cause contacting particles to become stuck into a rigid array. At a certain particle fraction $\phi^{*}>\phi_{\mathrm{c}}$ the necessity for collective motions becomes strong enough to cause a transition from a highly viscous fluid to a true solid for which the viscosity is infinite. The fraction $\phi^{*}$ can be considered equal to the maximum packing fraction $\phi_{\mathrm{cp}}=\pi / \sqrt{ } 18=0.7405$ for closepacked and $\phi_{\text {rcp }} \sim 0.64$ for random close-packed monodisperse spheres ${ }^{16}$ and decreases rapidly with increasing particle anisotropy. For the semidilute and concentrated regimes up to $\phi^{*}$ an asymptotic relationship between the relative viscosity $\eta_{\mathrm{r}}$ and $\phi$ was proposed by Krieger and Dougherty (so-called hard-sphere model $\left.^{51}\right)$ and is frequently used: ${ }^{23,36,37,40,55,56}$

$$
\eta_{\mathrm{r}}=\frac{\eta}{\eta_{\mathrm{HBP}}}=K\left(1-\frac{\phi}{\phi^{*}}\right)^{-\lambda}
$$

where $\eta$ and $\eta_{\text {HBP }}$ are the viscosities of the HBP/silica suspension and HBP, respectively, $K$ is related to the virial expansion of $\phi$ and varies between 0.88 and $1,{ }^{53}$ and the exponent $\lambda=[\eta] \phi^{*}$, where $[\eta]=2.5$ is the intrinsic viscosity for suspensions of spheres, ${ }^{57}$ or $\lambda=2$. The latter value, based on analogies between hydrodynamics and electrostatics, ${ }^{53}$ was used in the present work. Equation 2 is compared with the experimental data in Figure 9 using $\phi^{*}=$ $\phi_{\text {rcp }}=0.64$ and $K=1$ and is found to underestimate by far the measured increase in viscosity for the two types of $\mathrm{HBP} /$ silica suspensions.

To account for the actual increase in viscosity, an adsorbed and confined HBP layer on the surface of the particles was postulated. Immobilized layers were reported to increase the hydrodynamic

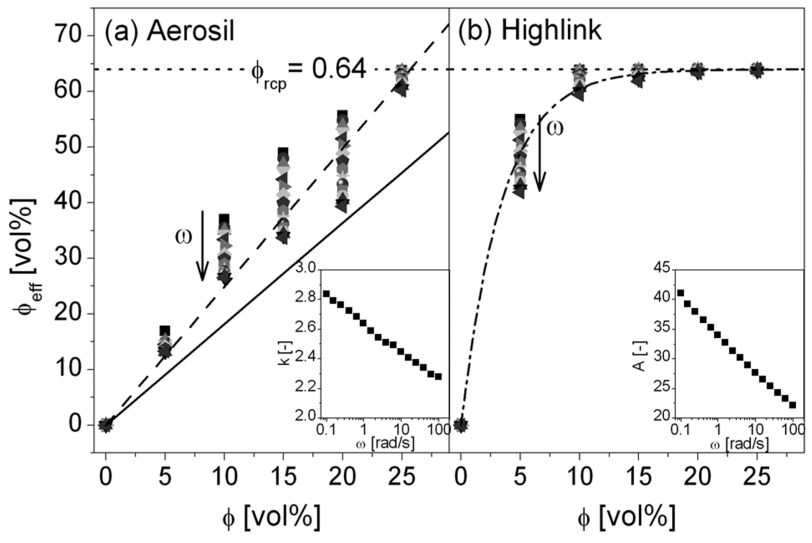

Figure 10. Effective particle volume fraction $\phi_{\mathrm{eff}}$ as a function of actual particle fraction $\phi$ calculated from suspension viscosity data using eq 2 with $K=1, \lambda=2$, and $\phi^{*}=0.64$, for frequencies $\omega$ in the range of $0.1-100 \mathrm{rad} / \mathrm{s}$. The solid line in (a) shows $\phi_{\text {eff }}$ calculated from the actual volume fraction of particles in Aerosil agglomerates (55\%). The dashed line in (a) is a fit of eq 3 at $\omega=6.28 \mathrm{rad} / \mathrm{s}$ with adjustable factor $k$; the dash-dotted line in (b) is a fit of eq 7 at $\omega=6.28 \mathrm{rad} / \mathrm{s}$ with adjustable factor $A$. The insets show the logarithmic dependence of the factors $k$ and $A$ on the frequency.

radius of the particles in proportion with the radius of gyration of the molecules (e.g., ref 23). The impact of an immobilized polymer layer on the rheological properties of polymer/particle suspensions was described in earlier studies. ${ }^{19,20,58}$ If one assumes that, under shear deformation, this layer moves with the particle, it has to be regarded as a contribution to the disperse phase; thus, it increases the solid volume fraction to an effective volume fraction $\phi_{\text {eff. }}{ }^{59,60}$ For a given immobilized layer thickness, the immobilized volume scales with the specific particle surface area and was expected to be larger for the Highlink case compared to the Aerosil case.

Equation 2 was inverted to calculate $\phi_{\text {eff }}$ for both types of suspensions from the experimental viscosity data (using $K=1$, $\lambda=2$, and $\phi^{*}=0.64$ ). The results are shown in Figure 10 for the investigated range of frequencies.

For Aerosil suspensions, $\phi_{\text {eff }}$ increased approximately linearly with $\phi$, reaching the packing limit $\phi^{*}=\phi_{\text {rcp }}=0.64$ at the highest investigated fraction $\phi=0.25$. The present $\phi_{\text {eff }}$ values are comparable with those obtained for various nanoparticle suspensions in PEG, depending on the molecular weight of the polymer. ${ }^{59,60}$ The large increase in effective volume was partially due to the fact that the agglomerates were not dense accumulations of particles, but diffuse aggregated structures of chemically bonded primary particles, where the space was infiltrated with HBP. Therefore, the agglomerate "particles" were considered as composites themselves with a $55 \%$ solid volume fraction. In other words, a suspension containing 5\% silica contained 9\% agglomerate particles. However, $\phi_{\text {eff }}$ was still higher than the calculated volume fraction of agglomerates (solid line in Figure 10a), and this was argued to result from an immobilized layer at the particle surface.

The effective volume fraction of Aerosil was considered to be proportional to the actual fraction:

$$
\phi_{\text {eff }}=k \phi
$$

where $k$ is a proportionality factor. Assuming that the immobilized layer formed a continuous shell of constant thickness $a$ on the surface of the particles, geometrical considerations led to the following expression for the effective volume fraction $\phi_{\text {eff }}$ :

$$
\phi_{\text {eff }}=\phi\left(1+\frac{a}{r}\right)^{3}
$$




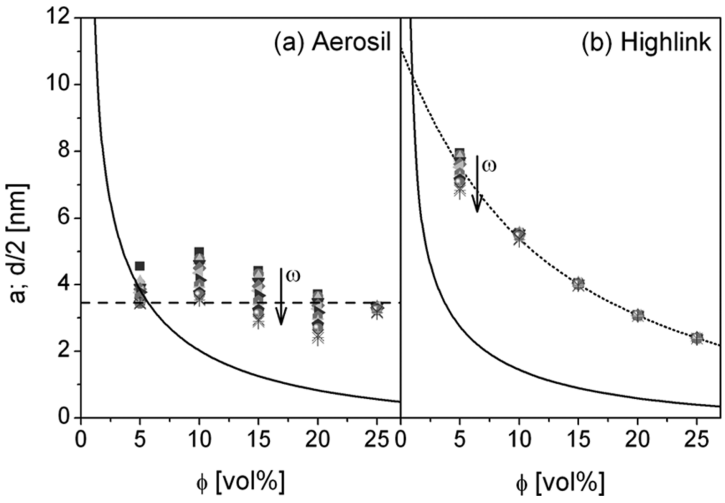

Figure 11. Immobilized layer thickness $a$ (symbols) and half of the mean free space between particles $d / 2$ (solid lines) as a function of (a) Aerosil (using $r=9.1 \mathrm{~nm}$ ) and (b) Highlink particle fraction $\phi$, for frequencies $\omega$ in the range of $0.1-100 \mathrm{rad} / \mathrm{s}$. The dashed line is the value of the immobilized layer thickness at $\omega=6.28 \mathrm{rad} / \mathrm{s}(a=3.3 \mathrm{~nm})$. The dotted line represents eq 9 at $\omega=6.28 \mathrm{rad} / \mathrm{s}$ (with $r=6.5 \mathrm{~nm}, \phi^{*}=$ 0.64 , and $A=29$ ).

where $r$ is the radius of the particles. In the case of Aerosil, two values of particle radius were considered: $6 \mathrm{~nm}$ (radius of the primary particles in the aggregates) and $9.1 \mathrm{~nm}$. The latter radius was calculated from the known specific surface $S\left(150 \mathrm{~m}^{2} / \mathrm{g}\right)$ and density $\rho\left(2.2 \mathrm{~g} / \mathrm{cm}^{3}\right)$ of the Aerosil powder as $3 /(\rho S)$.

Substituting $\phi$ by $\phi_{\text {eff }}$ in eq 2 (and using $K=1$ and $\lambda=2$ ) provides an expression for the relative viscosity of concentrated suspensions of particles surrounded by an immobilized layer:

$$
\eta_{\mathrm{r}}=\left(1-\frac{\phi}{\phi^{*}}\left(1+\frac{a}{r}\right)^{3}\right)^{-2}
$$

This approach disregards the fact that the Aerosil particles were agglomerated, which is discussed in a later paragraph. As also shown in Figure 10a, the factor $k$ was found to decrease linearly with $\log (\omega)$. Since $k$ is a function of particle size (eqs 3 and 4 ), this finding confirms the importance of particles on the emergence of the shear-thinning behavior of the suspensions visible in Figure 3. Equation 5 was fitted to the measured viscosity data at $\omega=6.28$ $\mathrm{rad} / \mathrm{s}$ with adjustable immobilized layer thickness $a$. The result shown in Figure 11 was obtained with $a=2.2 \mathrm{~nm}(r=6 \mathrm{~nm})$ or $a=3.3 \mathrm{~nm}(r=9.1 \mathrm{~nm})$. The fitted values of $a$ for all frequencies are shown in Figure 11a and found to lie in the range of 2-5 nm $(r=9.1 \mathrm{~nm})$, which is similar to the value of $5 \mathrm{~nm}$ found for PEO on silica particles. ${ }^{20}$ At $\phi_{\text {eff }}=\phi^{*}$, the layer thickness $a$ should be comparable to half of the mean free space $d$ between nearestneighbor particles, calculated according to Tewari et al.: ${ }^{61}$

$$
\begin{aligned}
d & =\left\langle H_{1}\right\rangle-2 r \\
& =r\left(K_{1}\left(\frac{3 \phi}{4 \pi}\right)^{-1 / 3}\left[1+B_{1}\left(\frac{\phi}{\phi_{\mathrm{cp}}}\right)^{2 / 3}\right]-2\right)
\end{aligned}
$$

where $\left\langle H_{1}\right\rangle$ is the distance between the cores of first nearestneighbor particles, $K_{1}=0.554, B_{1}=1.062$, and $\phi_{\mathrm{cp}}=\pi / \sqrt{ } 18$ is the highest volume fraction for close-packed spheres. Equation 6 should be considered as an approximation for the case where the particles are aggregated and not isolated spheres. The half distance $d / 2$ is plotted as a function of $\phi$ in Figure 11 and compared to the layer thickness values. For Aerosil suspensions the half distance between particles rapidly decreased with increasing particle fraction, whereas the layer thickness was roughly independent of volume fraction. In any case both values were of comparable magnitude, the differences resulting from the idealization of the actual heterogeneous microstructure shown in Figure 2.

In the case of the well-dispersed Highlink suspensions, $\phi_{\text {eff }}$ increased very rapidly with increasing $\phi$ (Figure $10 \mathrm{~b}$ ), reaching the packing limit $\phi^{*}=\phi_{\text {rcp }}=0.64$ at a fraction as low as $10 \%$. Similar results were reported for polyvinylidene fluoride nanocomposites. ${ }^{17,18}$ The dependence of the ratio $\phi_{\text {eff }} / \phi$ on $\phi$ invalidated the hard-sphere approach, and the effective particle fraction was fitted with an exponential form

$$
\phi_{\text {eff }}=\phi^{*}\left(1-\mathrm{e}^{-A \phi}\right)
$$

where the factor $A$ was found to be proportional to $\log (\omega)$ (inset in Figure 10b). Substituting eq 7 to the fraction $\phi$ in eq 2 (and using $K=1, \lambda=2$, and $\phi^{*}=0.64$ ) leads to an exponential dependence of the relative viscosity of Highlink suspensions on particle volume fraction:

$$
\eta_{\mathrm{r}}=e^{2 A \phi}
$$

Equation 8 was fitted to the measured viscosity data at $\omega=$ $6.28 \mathrm{rad} / \mathrm{s}$ with adjustable factor $A$ as shown in Figure 10.

As in the Aerosil case, the effective volume fraction $\phi_{\text {eff }}$ of the Highlink suspensions was related to the actual fraction $\phi$ through an immobilized layer of thickness $a$, assumed to fully cover each single silica particle of radius $r$ (eq 4). The value of $a$ was then calculated as a function of $\phi$ from the viscosity data at all investigated frequencies using eqs 4 and 7.

$$
a=r\left[\left(\frac{\phi^{*}\left(1-\mathrm{e}^{-A \phi}\right)}{\phi}\right)^{1 / 3}-1\right]
$$

As shown in Figure $11 \mathrm{~b}$ for $\omega=6.28 \mathrm{rad} / \mathrm{s}$, the layer thickness $a$ for the Highlink case decreased with filler fraction similarly to the distance between particles and was independent of frequency except at $5 \mathrm{vol} \%$ particle fraction. This result implies that it was the whole HBP volume, rather than a layer of finite thickness, which was immobilized. This is also why the calculated layer thickness was higher than the half distance. The idea of a gelled suspension at particle volume fraction larger than $5 \%$ is supported by the transition in $\tan (\delta)$, activation energy, and stress relaxation behavior shown in Figure 8 . The equilibrium thickness of the immobilized layer on the Highlink particles at a volume fraction of $5 \%$ was around $7.5 \mathrm{~nm}$.

An exponential dependence of the viscosity of suspensions on the particle volume fraction was first proposed by Arrhenius. ${ }^{62}$ It was suggested later by Thomas based on the work of Eyring. ${ }^{63,64}$ In the concentrated regime, a considerable rearrangement of particles must occur when the suspension is sheared and Eyring proposed an exponential form for the probability of a particle transfer from one shear plane to another. The exponential scaling was observed in various particle suspensions, ${ }^{65,66}$ which was argued to result from the deformable nature of the particles including the adsorbed layer so that the "hard-sphere" approach was no longer applicable. Interestingly, similar findings were reported for the low shear behavior of dendrimer solutions, ${ }^{67,68}$ gelling systems (with exponential scaling with cross-link density $^{69}$ ), and microgel suspensions. ${ }^{70,71}$ In this broad diversity of systems the exponential scaling was attributed to the deformable nature of the spherical molecules, with either a hard shell around a soft core (dendrimers) or a starlike microgel structure.

The exponential model thus appears to be specific of concentrated suspensions of particles with a shell of cross-linked molecules bound to the particle surface so that (i) they stay 


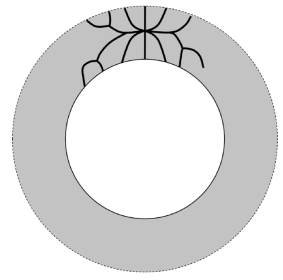

a)

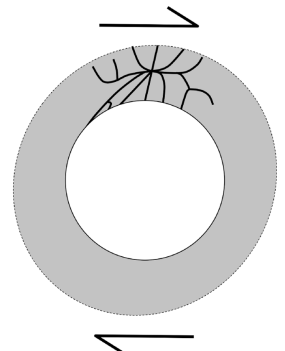

b)
Figure 12. Immobilized shell of cross-linked molecules on a silica particle at equilibrium (a) and under shear (b) leading to the observed exponential dependence of the viscosity of concentrated suspensions with particle volume fraction.

attached and (ii) show elastomer type deformation when the suspensions is sheared as sketched in Figure 12.

These two conditions were met in the case of the Highlink suspensions. The prevalent interaction between the silanol groups on the surface of the particles and the acrylated chain ends of the HBP was H-bonding, ${ }^{72}$ which was stronger than the intermolecular interactions between adjacent HBP molecules. H-bonds were observed for nanocomposites containing untreated silica particles and polymer matrices with carbonyl groups using various methods (FTIR, NMR, and DSC ${ }^{73,74}$ ). The deformation of the H-bonded HBP was also restricted if more than one branch was attached to the particle surface, leading to an elastomeric rather than a viscous response and self-similar dynamics (Figure 7). ${ }^{45}$ The average thickness of the shell was found to be equal to $7.5 \mathrm{~nm}$, which is comparable to the equilibrium radius of the single third generation HBP molecule $\left(4-5 \mathrm{~nm}^{75,76}\right)$. Since entanglement between such molecules can be disregarded, this result provides further evidence of the strong repulsive interactions resulting from the pinning of the molecules on the silica particles. ${ }^{23}$ One may also expect that, due to strong affinity of the HBP and the silanol surface of the silica particles, the globular HBP were squeezed together and align perpendicularly on the particle surface to form a dense starlike arrangement around the particles. The two conditions required for the exponential scaling were tested with suspensions of Highlink in DPHA. The affinity of this small starlike molecule with the silanol surface of the Highlink particles was similar to that of the acrylated HBP. The relative viscosity increase of Highlink suspensions in DPHA is depicted in Figure 9. In spite of limited amount of data, it is evident that the viscosity increased far beyond the hard-sphere prediction. The application of the exponential model led to a thinner immobilized shell $(3.5 \mathrm{~nm})$ than for the HBP, which again compares with the size of the smaller DPHA molecule.

If one of the above two conditions for the exponential scaling is not met, then the hard-sphere behavior is observed. This was the case for a number of suspensions with strong interfacial interactions, but which did not fulfill the second condition of crosslinking. ${ }^{20}$ This was also the case of the Aerosil suspensions in HBP, which did not fulfill the first condition of strong interactions since only intermolecular interactions were present between the methacrylate group of the silane (covalently attached to the silica surface ${ }^{77}$ ) and the acrylated chain ends of the HBP. The average thickness of the shell was found to be equal to $3.3 \mathrm{~nm}$. The relative viscosity of suspensions of Aerosil in DPHA is also shown in Figure 9 and found to be almost identical to that of the Aerosil/HBP case, with resulting shell thickness also close to $3.3 \mathrm{~nm}$. The coincidence of the relative viscosity of Aerosil suspensions in two different acrylate liquids, leading to the same shell thickness suggests that the methacrylsilane, rather than the dispersion state, was the main factor, which controlled the viscosity of the Aerosil suspensions.
The present work confirms the major influence of particle surface chemistry and resulting interfacial interactions on the rheological behavior of concentrated suspensions of nanoparticles. Further investigations of interfacial interactions in $\mathrm{HBP} /$ silica suspensions are ongoing and currently focus on calorimetric analysis similar to the study of Sargsyan et al. ${ }^{78}$ on poly(methyl methacrylate) $/ \mathrm{SiO}_{2}$ nanocomposites.

\section{Conclusions}

The rheological behavior of agglomerated and well-dispersed concentrated nanocomposite suspensions of silica in an acrylated HBP was investigated, using both untreated and silylated particles. Attention was paid to the influence of nanoparticle fraction on the viscoelastic properties, activation energy for viscous flow, and stress relaxation. $10 \mathrm{vol} \%$ of well-dispersed and untreated particles increased the HBP viscosity at $0.1 \mathrm{rad} / \mathrm{s}$ by more than 5 orders of magnitude. At $25 \mathrm{vol} \%$ the increase reached 8 orders of magnitude, whereas it was 1000 times less for agglomerated and silylated particles. The analysis of the experimental data systematically revealed the occurrence of a liquid-to-solid transition in the range of $20-25 \mathrm{vol} \%$ for agglomerated suspensions and in the range of 5-10 vol \% for the well-dispersed systems. In both cases the transition was lower than the maximum packing fraction for random suspensions. This was attributed to the presence of an immobilized polymer layer at the surface of the particles, resulting in an effective particle fraction higher than the actual fraction. Two rheological models were developed to predict the viscosity of the two types of concentrated suspensions as a function of filler fraction. In the case of agglomerated silanetreated silica with weak intermolecular interactions with the HBP, the asymptotic hard-sphere model established for concentrated suspensions was validated using an immobilized layer thickness in the range of $2-5 \mathrm{~nm}$. In the case of well-dispersed suspensions of untreated silica H-bonded with the HBP, the model predicted an exponential increase of the viscosity with particle fraction. In this case the whole HBP volume was found to be gelled when the particle volume fraction was larger than $5 \%$. The corresponding immobilized layer thickness was equal to $7.5 \mathrm{~nm}$.

Acknowledgment. Financial support from the Swiss National Science Foundation (SNF project \# 200020-119780) is gratefully acknowledged. Furthermore, the authors thank Henrik Bernquist from Perstorp AB for useful advice and the supply of samples.

\section{References and Notes}

(1) Brechet, Y.; Cavaille, J. Y. Y.; Chabert, E.; Chazeau, L.; Dendievel, R.; Flandin, L.; Gauthier, C. Adv. Eng. Mater. 2001, $3(8), 571-577$.

(2) Manias, E.; Touny, A.; Wu, L.; Strawhecker, K.; Lu, B.; Chung, T. C. Chem. Mater. 2001, 13 (10), 3516-3523.

(3) Mark, J. E. Acc. Chem. Res. 2006, 39 (12), 881-888.

(4) Bauer, F.; Glasel, H. J.; Decker, U.; Ernst, H.; Freyer, A.; Hartmann, E.; Sauerland, V.; Mehnert, R. Prog. Org. Coat. 2003, 47 (2), 147-153.

(5) Allaoui, A.; El Bounia, N. Express Polym. Lett. 2009, 3 (9), 588594

(6) Bokobza, L. Polymer 2007, 48 (17), 4907-4920.

(7) Xia, X. P.; Xie, C. S.; Cai, S. Z.; Wen, F.; Zhu, C. H.; Yang, X. L. Mater. Sci. Eng., A 2006, 429 (1-2), 329-333.

(8) Gomoll, A. H.; Fitz, W.; Scott, R. D.; Thornhill, T. S.; Bellare, A. Acta Orthop. 2008, 79 (3), 421-427.

(9) Pluta, M.; Galeski, A.; Alexandre, M.; Paul, M. A.; Dubois, P. J. Appl. Polym. Sci. 2002, 86 (6), 1497-1506.

(10) Zanetti, M.; Camino, G.; Reichert, P.; Mulhaupt, R. Macromol. Rapid Commun. 2001, 22 (3), 176-180.

(11) Fu, S. Y.; Feng, X. Q.; Lauke, B.; Mai, Y. W. Composites, Part B 2008, $39(6), 933-961$. 
(12) Wang, M.; Wang, W. Z.; Liu, T. X.; Zhang, W. D. Compos. Sci. Technol. 2008, 68 (12), 2498-2502.

(13) Cai, J. J.; Salovey, R. J. Polym. Sci., Polym. Phys. 1999, 37.

(14) Rodlert, M.; Plummer, C. J. G.; Leterrier, Y.; Månson, J.-A. E.; Grunbauer, H. J. M. J. Rheol. 2004, 48 (5), 1049-1065.

(15) Osman, M. A.; Atallah, A. Polymer 2006, 47 (7), 2357-2368.

(16) Anikeenko, A. V.; Medvedev, N. N. J. Struct. Chem. 2009, 50 (4), 761-768.

(17) Chandrasekhar, K. D.; Venimadhav, A.; Das, A. K. Appl. Phys. Lett. 2009, 95 (6), 3.

(18) Li, C. Y.; Chou, T. W. Appl. Phys. Lett. 2007, 90, 17.

(19) Sarvestani, A. S.; Jabbari, E. Macromol. Theory Simul. 2007, 16 (4), 378-385.

(20) Zhang, Q.; Archer, L. A. Langmuir 2002, 18 (26), 10435-10442.

(21) Goertzen, W. K.; Sheng, X.; Akinc, M.; Kessler, M. R. Polym. Eng. Sci. 2008, 48 (5), 875-883.

(22) Cohenaddad, J. P. Polymer 1989, 30 (10), 1820-1823.

(23) Anderson, B. J.; Zukoski, C. F. Macromolecules 2008, 41 (23), 9326-9334.

(24) Frechet, J. M. J.; Hawker, C. J.; Gitsov, I.; Leon, J. W. J. Macromol. Sci., Pure Appl. Chem. 1996, A33 (10), 1399-1425.

(25) Bosman, A. W.; Janssen, H. M.; Meijer, E. W. Chem. Rev. 1999, 99 (7), 1665-1688.

(26) Plummer, C. J. G.; Luciani, A.; Nguyen, T. Q.; Garamszegi, L.; Rodlert, M.; Månson, J.-A. E. Polym. Bull. 2002, 49 (1), 77-84.

(27) Kim, Y. H. J. Polym. Sci., Polym. Chem. 1998, 36 (11), 1685-1698.

(28) Voit, B. I. Acta Polym. 1995, 46 (2), 87-99.

(29) Boogh, L.; Pettersson, B.; Månson, J.-A. E. Polymer 1999, 40 (9), 2249-2261.

(30) Mezzenga, R.; Boogh, L.; Månson, J.-A. E. Compos. Sci. Technol. 2001, 61 (5), 787-795.

(31) Klee, J. E.; Schneider, C.; Holter, D.; Burgath, A.; Frey, H.; Mulhaupt, R. Polym. Adv. Technol. 2001, 12 (6), 346-354.

(32) Schmidt, L. E.; Schmah, D.; Leterrier, Y.; Månson, J.-A. E. Rheol. Acta 2007, 46 (5), 693-701.

(33) Schmidt, L. E.; Yi, S.; Jin, Y. H.; Leterrier, Y.; Cho, Y. H.; Månson, J.-A. E. J. Micromech. Microeng. 2008, 18 (4), 45022.

(34) Geiser, V.; Leterrier, Y.; Månson, J.-A. E. J. Appl. Polym. Sci. 2009, 114 (3), 1954-1963.

(35) Percy, M. J.; Amalvy, J. I.; Randall, D. P.; Armes, S. P.; Greaves, S. J.; Watts, J. F. Langmuir 2004, 20 (6), 2184-2190.

(36) Dekruif, C. G.; Vanlersel, E. M. F.; Vrij, A.; Russel, W. B. J. Chem. Phys. 1985, 83 (9), 4717-4725.

(37) Cheng, Z. D.; Zhu, J. X.; Chaikin, P. M.; Phan, S. E.; Russel, W. B. Phys. Rev. E 2002, 65 (4), 041405.

(38) Bossis, G.; Brady, J. F. J. Chem. Phys. 1989, 91 (3), 1866-1874.

(39) Bender, J.; Wagner, N. J. J. Rheol. 1996, 40 (5), 899-916.

(40) Foss, D. R.; Brady, J. F. J. Fluid Mech. 2000, 407, 167-200.

(41) Laun, H. M.; Bung, R.; Schmidt, F. J. Rheol. 1991, 35 (6), 999-1034.

(42) Raghavan, S. R.; Khan, S. A. J. Colloid Interface Sci. 1997, 185 (1), 57-67.

(43) Lin, C. R.; Chen, W. J. Colloid Polym. Sci. 1999, 277 (11), 10191025.

(44) Nguyen, Q. D.; Boger, D. V. Annu. Rev. Fluid Mech. 1992, 24, 47-88.

(45) Curro, J. G.; Pincus, P. Macromolecules 1983, 16 (4), 559-562.
(46) Kapnistos, M.; Lang, M.; Vlassopoulos, D.; Pyckhout-Hintzen, W.; Richter, D.; Cho, D.; Chang, T.; Rubinstein, M. Nature Mater. 2008, 7 (12), 997-1002.

(47) Plummer, C. J. G.; Garamszegi, L.; Leterrier, Y.; Rodlert, M.; Månson, J.-A. E. Chem. Mater. 2002, 14 (2), 486-488.

(48) Jing, D. Y.; Ding, J. D. Macromol. Biosci. 2007, 7 (12), 1290-1298.

(49) Einstein, A. Ann. Phys. 1911, 34 (3), 591-592.

(50) Bicerano, J.; Douglas, J. F.; Brune, D. A. J. Macromol. Sci., Rev. Macromol. Chem. Phys. 1999, C39 (4), 561-642.

(51) Krieger, I. M. Adv. Colloid Interface 1972, 3 (2), 111-136.

(52) Ball, R. C.; Richmond, P. Phys. Chem. Liq. 1980, 9 (2), 99-116.

(53) Douglas, J. F.; Garboczi, E. J., Adv. Chem. Phys. 1995, 91, 85-153.

(54) Russel, W. B. J. Chem. Soc., Faraday Trans. 2 1984, 80, 31-41.

(55) Brady, J. F. J. Chem. Phys. 1993, 99 (1), 567-581.

(56) Phan, S. E.; Russel, W. B.; Cheng, Z. D.; Zhu, J. X.; Chaikin, P. M.; Dunsmuir, J. H.; Ottewill, R. H. Phys. Rev. E 1996, 54 (6), 6633-6645.

(57) Krieger, I. M.; Dougherty, T. J. Trans. Soc. Rheol. 1959, 3, $137-152$.

(58) Windhab, E. J. Appl. Rheol. 2000, 10 (3), 134-144.

(59) Anderson, B. J.; Zukoski, C. F. Macromolecules 2009, 42 (21), $8370-8384$

(60) Vickers, D.; Archer, L. A.; Floyd-Smith, T. Colloids Surf., A 2009, $348(1-3), 39-44$.

(61) Tewari, A.; Gokhale, A. M. Mater. Sci. Eng., A 2004, 385 (1-2), 332-341.

(62) Arrhenius, S. Z. Phys. Chem. 1887, 1, 285.

(63) Thomas, D. G. J. Colloid Sci. 1965, 20, 3, 267.

(64) Eyring, H.; Henderson, D.; Stover, B. J.; Eyring, E. M. Statistical Mechanics and Dynamics; Wiley: New York, 1964.

(65) Das Kanungo, J. L.; McAtee, J. L. Appl. Clay Sci. 1986, 1, 285.

(66) Hone, J. H. E.; Howe, A. M. J. Colloid Interface Sci. 2002, 251 (1), 193-199.

(67) Uppuluri, S.; Keinath, S. E.; Tomalia, D. A.; Dvornic, P. R. Macromolecules 1998, 31 (14), 4498-4510.

(68) Rietveld, I. B.; Bedeaux, D. J. Colloid Interface Sci. 2001, 235 (1), 89-92.

(69) Kheirandish, S.; Gubaydullin, I.; Willenbacher, N. Rheol. Acta 2009, 48 (4), 397-407.

(70) Borrega, R.; Cloitre, M.; Betremieux, I.; Ernst, B.; Leibler, L. Europhys. Lett. 1999, 47 (6), 729-735.

(71) Senff, H.; Richtering, W. Colloid Polym. Sci. 2000, 278 (9), 830840

(72) Sideridou, I. D.; Karabela, M. M. J. Appl. Polym. Sci. 2008, 110 (1), 507-516.

(73) Blum, F. D.; Krisanangkura, P. Thermochim. Acta 2009, 492 (1-2), 55-60.

(74) Metin, B.; Blum, F. D. Langmuir 2010, 26 (7), 5226-5231.

(75) Gusev, S. I.; Zaitsev, S. D.; Semchikov, Y. D.; Zakharova, O. G. Russ. J. Appl. Chem. 2006, 79 (8), 1338-1340.

(76) Boogh, L.; Jannerfeldt, G.; Pettersson, B.; Björnberg, H.; Månson, J.-A. E. Proceedings of the ICCM-12 Conference, Paris, France, July 5-9, 1999.

(77) Ishida, H.; Miller, J. D. Macromolecules 1984, 17 (9), 1659-1666.

(78) Sargsyan, A.; Tonoyan, A.; Davtyan, S.; Schick, C. Eur. Polym. J. 2007, 43 (8), 3113-3127. 